\title{
A pan-Canadian prospective study of young women with breast cancer: the rationale and protocol design for the RUBY study
}

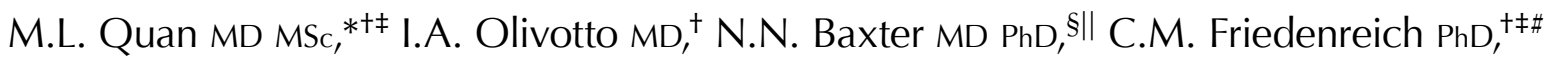 \\ K. Metcalfe RN PhD, ${ }^{* *}$ E. Warner MD MSc, ${ }^{+\dagger}$ K. MacLennan MSc, ${ }^{\neq \neq}$J.E. Stephen $\mathrm{PhD}_{1}{ }^{\S}$ \\ M.R. Akbari $M D, \| \# \#$ D. Howell RN PhD, \# S. Narod MD, $\|^{\| \#}$ and the RUBY site investigators
}

\begin{abstract}
Introduction The understanding of the biology and epidemiology of, and the optimal therapeutic strategies for, breast cancer ( $\mathrm{BCa}$ ) in younger women is limited. We present the rationale, design, and initial recruitment of Reducing the Burden of Breast Cancer in Young Women (RUBY), a unique national prospective cohort study designed to examine the diagnosis, treatment, quality of life, and outcomes from the time of diagnosis for young women with BCa.

Methods Over a 4-year period at 33 sites across Canada, the RUBY study will use a local and virtual recruitment model to enrol 1200 women with BCa who are 40 years of age or younger at the time of diagnosis, before initiation of any treatment. At a minimum, comprehensive patient, tumour, and treatment data will be collected to evaluate recurrence and survival. Patients may opt to complete patient-reported questionnaires, to provide blood and tumour samples, and to be contacted for future research, forming the core dataset from which 4 subprojects evaluating genetics, lifestyle factors, fertility, and local management or delivery of care will be performed.
\end{abstract}

Summary The RUBY study will be the most comprehensive repository of data, biospecimens, and patient-reported outcomes ever collected with respect to young women with BCa from the time of diagnosis, enabling research unique to that population now and into the future. This research model could be used for other oncology settings in Canada.

Key Words Breast cancer, young-onset; prospective cohorts; pan-Canadian studies; knowledge translation; patient-reported outcomes; biorepositories

Curr Oncol. 2020 October27(5)e516-e523

www.current-oncology.com

\section{INTRODUCTION}

In 2019, an estimated 26,900 Canadian women received a breast cancer (BCa) diagnosis and 5000 died from the disease $^{1}$. Women 40 years of age and younger account for approximately $5 \%$ of BCa cases and are more likely to be diagnosed with later-stage disease and to have biologically aggressive phenotypes, more recurrences, and greater mortality ${ }^{2-4}$. Despite known differences in tumour phenotypes and outcomes in young compared with older women, the understanding of the biology and epidemiology of, and the optimal therapeutic strategies for, BCa in young women is limited. In addition, very little patient-reported experience and outcomes data have been generated, despite the unique challenges that patients face given their life stage and age. Although cohort studies of young women with BCa such as the POSH $^{5}$ and Harvard ${ }^{6}$ cohorts have been created, they did not recruit patients at the time of diagnosis, before treatment initiation, resulting in a knowledge gap for that critical period.

To address those issues and others, we designed the Reducing the Burden of Breast Cancer in Young Women (RUBY) study, a prospective cohort of young women recruited from a variety of practice settings across Canada at the time of their BCa diagnosis. In addition, we created a network of clinicians and researchers involved with delivery of BCa care, providing infrastructure for health systems research and knowledge translation of findings into practice.

This paper details the methods of the RUBY cohort study to illustrate the feasibility of oncology research in the 
real-world setting for a unique subpopulation of patients. The RUBY methods could be translatable to other diseases with similar research challenges.

\section{METHODS}

\section{Study Design}

The RUBY study is a prospective cohort study of patients newly diagnosed with BCa who are 40 years of age and younger. The goal is to recruit 1200 women from diverse Canadian settings, including non-academic sites. Enrolment in RUBY requires, at a minimum, consent to collect extensive chart review data to confirm details of diagnosis, treatment, and oncologic outcomes such as recurrence and death, the primary study outcomes. The second level of participation consists of online questionnaires, providing patient-reported outcomes measures, including factors previously not evaluable in this cohort (Table I). The third level of participation requires donation of biospecimens. Blood samples are taken before initiation of the 1st primary treatment and at years 1,2, and 3, permitting further analysis of biomarkers, including hormones, inflammatory markers, micronutrient levels, and fertility measures over time. Germline mutation testing for common genes such as BRCA1/2 and a panel of 23 genes (BROCAP, Table II) having known associations with BCa is done, as is whole-genome mapping. Tumour samples from surgical pathology specimens are processed into tissue microarrays for future molecular analysis. Finally, patients are asked for permission for future contact for research purposes. The patients are enrolled at the time of initial surgical consultation, after diagnosis, but before their first treatment (surgery or systemic therapy).

This core RUBY cohort data and biospecimen repository will serve to provide the infrastructure for 4 initial subprojects focusing on the role of genetics (KM), lifestyle and modifiable risk factors (CMF), fertility (EW), and local therapies or delivery of care (NNB), the details of which are described elsewhere-in addition to future studies.

\section{Study Population}

Women newly diagnosed with histologically-confirmed BCa are eligible to participate if they

are diagnosed with invasive $\mathrm{BCa}$, ductal carcinoma in situ, or malignant phyllodes tumour in Canada.

are 18 or more years of age and have not yet reached their 41st birthday.

are able to provide written informed consent and to complete questionnaires in English or French.

\section{Recruitment}

The recruitment sites (Table III) were selected to represent a variety of clinical practice settings. Sites with women from inner city, rural, Indigenous, and immigrant populations were cultivated, and provisions to support recruitment within those populations were enacted. The RUBY sites account for most of the BCa surgical treatment in their respective regions.

Each site identifies potentially eligible participants at initial referral to a surgeon once a diagnosis of malignancy is known. Eligible patients are flagged so that clinic staff can introduce them to the study and seek verbal consent for direct contact by the RUBY study team. Two recruitment models are being used to accommodate local resources and capacity. The "local" (LRC) model is implemented at centres with available institutional research assistants or other personnel. The "virtual" (VRC) model is used at centres without available research resources, such that sites refer electronically, and the VRC located in Calgary contacts the patients directly by telephone or e-mail. The LRC or VRC is responsible for recruitment, consent, guidance for questionnaire completion, blood draws, and medical chart data abstraction. Eligibility or screening logs are maintained at each site. To optimize enrolment, e-mail reminder messages and study updates are sent at regular intervals.

\section{Data Collection}

Participants complete questionnaires online using the REDCap (Research Electronic Data Capture: REDCap Consortium, Vanderbilt University, Nashville, TN, U.S.A.) tool hosted at the Applied Health Research Centre (AHRC) at St. Michael's Hospital in Toronto, Ontario. Active follow-up for treatment and cancer outcomes with participant online questionnaires and chart abstraction will be conducted for up to 3 years. Passive follow-up will be conducted annually by regular vital status linkages and individual chart abstractions to identify dates and sites of any one or more of progression, recurrence, or new primary cancers. National and provincial administrative databases will be accessed through provincial patient health numbers to ascertain medical interventions, vital status, and if applicable, date and cause of death. Figure 1 depicts the flow through the study.

\section{Patient Questionnaires}

A link to access online questionnaires from the RUBY REDCap database is sent to participants by e-mail shortly after enrolment. Participants are asked to complete questionnaires at baseline (shortly after diagnosis, but before treatment), at 3 months, after initial active treatment (roughly 6-9 months), and annually for 3 years after enrolment. Table I summarizes the questionnaires requested for completion at each time point. The full set of instruments includes more than 2500 questions contained in more than 40 surveys.

\section{Medical Record Abstraction}

All diagnosis, staging, treatment, and follow-up care data are obtained through medical chart abstraction using a standardized abstraction tool and data dictionary. Either the LRC or the VRC conducts the chart abstraction at years 1 and 3. Relevant records are obtained from each institution's health records department or the participant's electronic medical record and are sent to the central coordinating centre at yearly intervals. All data are entered into the RUBY database by the LRCs or the VRC using the Web-based Medidata Rave application (3DS, Paris, France) housed at the AHRC in Toronto. Relevant medical variables, including all tumour and treatment data, are abstracted, as are oncologic outcomes including recurrence, progression, and death. We 
TABLE I Questionnaires for participant completion

\begin{tabular}{|c|c|c|c|c|c|c|}
\hline \multirow{3}{*}{$\begin{array}{l}\text { Questionnaire } \\
\text { group }\end{array}$} & \multicolumn{6}{|c|}{ Study time point } \\
\hline & \multirow[t]{2}{*}{ Baseline } & \multirow[t]{2}{*}{3 Months } & \multirow{2}{*}{$\begin{array}{l}\text { Post treatment } \\
\text { ( } 6 \text { or } 9 \text { months) }\end{array}$} & \multicolumn{3}{|c|}{ Post diagnosis } \\
\hline & & & & 1 Year & 2 Years & 3 Years \\
\hline \multicolumn{7}{|c|}{ Health questionnaires-module 1} \\
\hline & $\begin{array}{l}\text { Breast cancer diagnosis } \\
\text { and treatment }\end{array}$ & Fertility & $\begin{array}{l}\text { Breast surgery } \\
\text { and reconstruction }\end{array}$ & Annual fertility & Annual fertility & Annual fertility \\
\hline & $\begin{array}{l}\text { Breast imaging and } \\
\text { surgeries }\end{array}$ & $\begin{array}{l}\text { Treatment } \\
\text { plan }\end{array}$ & & Annual genetics & Annual genetics & Annual genetics \\
\hline & \multicolumn{6}{|l|}{$\begin{array}{l}\text { Menstrual and } \\
\text { reproductive history }\end{array}$} \\
\hline & \multicolumn{6}{|l|}{ Birth control history } \\
\hline & \multicolumn{6}{|l|}{ Medical history } \\
\hline & Family history & & & $\begin{array}{l}\text { Medical history } \\
\text { follow-up }\end{array}$ & $\begin{array}{l}\text { Medical history } \\
\text { follow-up }\end{array}$ & $\begin{array}{l}\text { Medical history } \\
\text { follow-up }\end{array}$ \\
\hline & Demographics & & & & & \\
\hline
\end{tabular}

Patient-reported outcomes-module 2

\begin{tabular}{|c|c|c|c|c|}
\hline Breast-Q $Q^{12}$ & Breast-Q & Breast-Q & Breast-Q & Breast-Q \\
\hline $\begin{array}{l}\text { Cancer Behaviour } \\
\text { Inventory }^{13} \text { (CBI-B) }\end{array}$ & CBI-B & $\begin{array}{l}\text { Cancer Survivor } \\
\text { Self-Efficacy Scale }\end{array}$ & $\begin{array}{l}\text { Cancer Survivor } \\
\text { Self-Efficacy Scale }\end{array}$ & $\begin{array}{l}\text { Cancer Survivor } \\
\text { Self-Efficacy Scale }\end{array}$ \\
\hline General anxiety $^{14}$ & General anxiety & General anxiety & General anxiety & General anxiety \\
\hline Feelings of depression ${ }^{15}$ & $\begin{array}{l}\text { Feelings } \\
\text { of depression }\end{array}$ & $\begin{array}{l}\text { Feelings } \\
\text { of depression }\end{array}$ & $\begin{array}{c}\text { Feelings } \\
\text { of depression }\end{array}$ & $\begin{array}{c}\text { Feelings } \\
\text { of depression }\end{array}$ \\
\hline PHQ-916 & PhQ-9 & PhQ-9 & PhQ-9 & PhQ-9 \\
\hline Trauma Checklist $^{17}$ & Trauma Checklist & Trauma Checklist & Trauma Checklist & Trauma Checklist \\
\hline \multicolumn{5}{|l|}{ Personality Survey (IPIP) ${ }^{18}$} \\
\hline $\begin{array}{l}\text { Pittsburgh Sleep Quality } \\
\text { Index }\end{array}$ & $\begin{array}{c}\text { Pittsburgh } \\
\text { Sleep Quality Index }\end{array}$ & $\begin{array}{c}\text { Pittsburgh } \\
\text { Sleep Quality Index }\end{array}$ & $\begin{array}{c}\text { Pittsburgh } \\
\text { Sleep Quality Index }\end{array}$ & $\begin{array}{c}\text { Pittsburgh } \\
\text { Sleep Quality Index }\end{array}$ \\
\hline \multirow[t]{6}{*}{ Marital Adjustment ${ }^{20}$} & & Marital Adjustment & Marital Adjustment & Marital Adjustment \\
\hline & $\begin{array}{l}\text { Reproductive } \\
\text { concerns after } \\
\text { cancer }\end{array}$ & $\begin{array}{l}\text { Reproductive } \\
\text { concerns after } \\
\text { cancer }\end{array}$ & $\begin{array}{l}\text { Reproductive } \\
\text { concerns after } \\
\text { cancer }\end{array}$ & $\begin{array}{l}\text { Reproductive } \\
\text { concerns after } \\
\text { cancer }\end{array}$ \\
\hline & $\begin{array}{c}\text { Use of } \\
\text { psychological } \\
\text { and CAM therapies }\end{array}$ & $\begin{array}{c}\text { Use of } \\
\text { psychological } \\
\text { and CAM therapies }\end{array}$ & $\begin{array}{c}\text { Use of } \\
\text { psychological } \\
\text { and CAM therapies }\end{array}$ & $\begin{array}{c}\text { Use of } \\
\text { psychological } \\
\text { and CAM therapies }\end{array}$ \\
\hline & & $\begin{array}{l}\text { Fear of cancer } \\
\text { recurrence }\end{array}$ & $\begin{array}{l}\text { Fear of cancer } \\
\text { recurrence }\end{array}$ & $\begin{array}{l}\text { Fear of cancer } \\
\text { recurrence }\end{array}$ \\
\hline & $\begin{array}{l}\text { Illness Intrusiveness } \\
\text { Rating scale }\end{array}$ & $\begin{array}{l}\text { Illness Intrusiveness } \\
\text { Rating scale }\end{array}$ & $\begin{array}{l}\text { Illness Intrusiveness } \\
\text { Rating scale }\end{array}$ & $\begin{array}{l}\text { Illness Intrusiveness } \\
\text { Rating scale }\end{array}$ \\
\hline & & Work situation & Work situation & Work situation \\
\hline $\begin{array}{l}\text { MOS-Social Support } \\
\quad(\text { MOS-SS })^{21}\end{array}$ & MOS-SS & MOS-SS & MOS-SS & MOS-SS \\
\hline $\begin{array}{l}\text { Cancer Fatigue Scale } \\
(\text { CFS })^{22}\end{array}$ & CFS & CFS & CFS & CFS \\
\hline \multirow[t]{2}{*}{$\begin{array}{c}\text { Breast Cancer Prevention } \\
\text { Trial Symptom Checklist } \\
\text { (BCPT) }\end{array}$} & ВСРТ & ВСРТ & ВСРТ & ВСРТ \\
\hline & $\begin{array}{l}\text { Adherence-related } \\
\text { measures }\end{array}$ & $\begin{array}{l}\text { Adherence-related } \\
\text { measures }\end{array}$ & $\begin{array}{l}\text { Adherence-related } \\
\text { measures }\end{array}$ & $\begin{array}{l}\text { Adherence-related } \\
\text { measures }\end{array}$ \\
\hline Beliefs about medicines & $\begin{array}{c}\text { Beliefs } \\
\text { about medicines }\end{array}$ & $\begin{array}{c}\text { Beliefs } \\
\text { about medicines }\end{array}$ & $\begin{array}{c}\text { Beliefs } \\
\text { about medicines }\end{array}$ & $\begin{array}{c}\text { Beliefs } \\
\text { about medicines }\end{array}$ \\
\hline
\end{tabular}


TABLE I Continued

\begin{tabular}{|c|c|c|c|c|c|c|}
\hline \multirow{3}{*}{$\begin{array}{l}\text { Questionnaire } \\
\text { group }\end{array}$} & \multicolumn{6}{|c|}{ Study time point } \\
\hline & \multirow[t]{2}{*}{ Baseline } & \multirow[t]{2}{*}{3 Months } & \multirow{2}{*}{$\begin{array}{l}\text { Post treatment } \\
\text { ( } 6 \text { or } 9 \text { months) }\end{array}$} & \multicolumn{3}{|c|}{ Post diagnosis } \\
\hline & & & & 1 Year & 2 Years & 3 Years \\
\hline & $\begin{array}{l}\text { Side-effect } \\
\text { expectations }\end{array}$ & & $\begin{array}{c}\text { Side-effect } \\
\text { expectations }\end{array}$ & $\begin{array}{l}\text { Side-effect } \\
\text { expectations }\end{array}$ & & \\
\hline & $\begin{array}{l}\text { Brief Illness Perception } \\
\text { Questionnaires (BIPQ) }^{23}\end{array}$ & & BIPQ & BIPQ & BIPQ & BIPQ \\
\hline & & & & $\begin{array}{l}\text { Decision Regret } \\
\text { Scale }\end{array}$ & $\begin{array}{l}\text { Decision Regret } \\
\text { Scale }\end{array}$ & $\begin{array}{c}\text { Decision Regret } \\
\text { Scale }\end{array}$ \\
\hline \multicolumn{7}{|c|}{ Lifestyle-module 3} \\
\hline \multicolumn{7}{|c|}{ Lifestyle } \\
\hline & $\begin{array}{l}\text { Physical Activity and } \\
\text { Sedentary Behaviour } \\
\text { Questionnaire }{ }^{11}\end{array}$ & & & $\begin{array}{l}\text { Physical Activity } \\
\text { and Sedentary } \\
\text { Behaviour } \\
\text { Questionnaire }\end{array}$ & & $\begin{array}{c}\text { Physical Activity } \\
\text { and Sedentary } \\
\text { Behaviour } \\
\text { Questionnaire }\end{array}$ \\
\hline & $\begin{array}{l}\text { Food Frequency } \\
\text { Questionnaire }^{10}\end{array}$ & & & $\begin{array}{l}\text { Food Frequency } \\
\text { Questionnaire }\end{array}$ & & $\begin{array}{c}\text { Food Frequency } \\
\text { Questionnaire }\end{array}$ \\
\hline \multicolumn{7}{|c|}{ Total time commitment } \\
\hline & 2-2.5 Hours & 10 Minutes & 45-60 Minutes & 1.5-2 Hours & 45-60 Minutes & 1.5-2 Hours \\
\hline
\end{tabular}

TABLE II The known breast (BCa) and ovarian cancer (OCa) predisposing genes and their characteristics by risk category

\begin{tabular}{|c|c|c|c|c|c|c|c|}
\hline Gene & Chromosome & Gene & Chromosome & Gene & Chromosome & Gene & Chromosome \\
\hline ATM & 11 & EPCAM & 2 & $N B N$ & 8 & RAD51D & 17 \\
\hline$B A R D 1$ & 2 & FAM175A & 4 & PALB2 & 16 & STK11 & 19 \\
\hline$B R C A 1$ & 17 & MLH1 & 3 & PPM1D & 17 & TP53 & 17 \\
\hline$B R C A 2$ & 13 & MRE11A & 11 & PMS2 & 7 & $X R C C 2$ & 7 \\
\hline$B R I P 1$ & 17 & $\mathrm{MSH} 2$ & 2 & PTEN & 10 & & \\
\hline $\mathrm{CDH} 1$ & 16 & MSH6 & 2 & RAD50 & 5 & & \\
\hline CHEK2 & 22 & MUTYH & 1 & RAD51C & 17 & & \\
\hline \multicolumn{2}{|c|}{ Characteristic } & \multicolumn{2}{|c|}{ High risk } & \multicolumn{2}{|c|}{ Moderate risk } & \multicolumn{2}{|c|}{ Newer } \\
\hline \multicolumn{2}{|l|}{ Symbol } & \multicolumn{2}{|c|}{$\begin{array}{c}\text { BRCA1, BRCA2, CDH1, } \\
\text { EPCAM, PMS2, PTEN, MLH1, } \\
\text { MSH2, MSH6, STK11, TP53 }\end{array}$} & \multicolumn{2}{|c|}{ ATM, CHEK2, PALB2 } & \multicolumn{2}{|c|}{$\begin{array}{c}\text { BARD1, BRIP1, FAM175A } \\
\text { (ABRAXAS), MRE11A, MUTYH, } \\
\text { NBN, RECQL, RAD50, } \\
\text { RAD51C, RAD51D, XRCC2 }\end{array}$} \\
\hline \multicolumn{2}{|c|}{$\begin{array}{l}\text { Associated risk } \\
\text { for BCa or OCa }\end{array}$} & \multicolumn{2}{|c|}{$\begin{array}{l}\text { Significantly increased risk } \\
\text { (usually at least } 4 x \text {, often more) }\end{array}$} & \multicolumn{2}{|c|}{$\begin{array}{l}\text { Moderately increased risk } \\
\text { (approximately } 2-4 x \text { ) }\end{array}$} & \multicolumn{2}{|c|}{$\begin{array}{c}\text { Exact lifetime risk } \\
\text { not yet known }\end{array}$} \\
\hline \multicolumn{2}{|c|}{ Risk for other cancers? } & \multicolumn{2}{|c|}{ Can increase the risk } & \multicolumn{2}{|c|}{ Can increase the risk } & \multicolumn{2}{|c|}{ Can increase the risk } \\
\hline \multicolumn{2}{|c|}{ Well studied? } & \multicolumn{2}{|c|}{ Yes } & \multicolumn{2}{|c|}{ Yes } & \multicolumn{2}{|c|}{ Not as well } \\
\hline \multicolumn{2}{|c|}{$\begin{array}{l}\text { Guidelines for screening } \\
\text { and prevention? }\end{array}$} & \multicolumn{2}{|c|}{ Defined guidelines available } & \multicolumn{2}{|c|}{$\begin{array}{c}\text { No defined guidelines } \\
\text { yet available }\end{array}$} & \multicolumn{2}{|c|}{$\begin{array}{l}\text { No defined guidelines } \\
\text { yet available }\end{array}$} \\
\hline
\end{tabular}

previously used established data collection methods to retrospectively collect those data elements at a population level in Ontario, Alberta, British Columbia, and Quebec ${ }^{7-9}$.

\section{Blood Sample Collection}

All participants consenting to provide blood samples are provided requisitions and have their blood drawn at designated laboratories. Local clinical labs were selected at each site to minimize extra visits for patients; they include hospital-based labs, research-nurse draws during clinical encounters, and provincial or group lab contracts (LifeLabs, Ontario; Alberta Precision Laboratories, Alberta). Each laboratory collects and processes blood samples according to the RUBY blood protocol (Figure 2). Samples are shipped to the central biobank at Women's College Hospital semi-annually. 
TABLE III Recruitment sites in RUBY active as of January 2019 (exceptions denoted with an asterisk)

\begin{tabular}{|c|c|}
\hline 1 & BC Cancer, Vancouver, BC* \\
\hline 2 & Calgary Breast Health Program, Calgary, AB \\
\hline 3 & Cancer Centre of Southeastern Ontario, Kingston, ON \\
\hline 4 & $\begin{array}{l}\text { Centre des maladies du sein Deschênes-Fabia, Quebec City, } \\
\text { QC }\end{array}$ \\
\hline 5 & CHUM Breast Cancer Clinic, Montreal, QC \\
\hline 6 & $\begin{array}{l}\text { Edmonton Comprehensive Breast Care Program, Edmonton, } \\
\text { AB }\end{array}$ \\
\hline 7 & Guelph General Hospital, Guelph, ON \\
\hline 8 & IWK Health Centre, Halifax, NS \\
\hline 9 & Sir Mortimer B. Davis Jewish General Hospital, Montreal, QC \\
\hline 10 & Juravinski Cancer Centre, Hamilton, ON \\
\hline 11 & Irene and Leslie Dubé Centre of Care, Saskatoon, SK \\
\hline 12 & Lethbridge Breast Health Program, Lethbridge, $A B$ \\
\hline 13 & London Breast Clinic, London, ON \\
\hline 14 & Hôpital Maisonneuve-Rosemont, Montreal, QC \\
\hline 15 & McGill University Health Centre, Montreal, QC \\
\hline 16 & Nanaimo Regional General Hospital, Nanaimo, BC \\
\hline 17 & Dr. H. Bliss Murphy Cancer Centre, St John's, NL \\
\hline 18 & $\begin{array}{l}\text { North York General Hospital Breast Diagnostic Clinic, } \\
\text { Toronto, ON }\end{array}$ \\
\hline 19 & Princess Margaret Hospital, Toronto, ON \\
\hline 20 & Providence Breast Centre, Vancouver, BC \\
\hline 21 & Sault Area Hospital, Sault Ste. Marie, ON \\
\hline 22 & $\begin{array}{l}\text { Sindi Ahluwalia Hawkins Centre for the Southern Interior, } \\
\text { Kelowna, BC }\end{array}$ \\
\hline 23 & Simcoe Muskoka Regional Cancer Centre, Barrie, ON \\
\hline 24 & St. Michael's Hospital, Toronto, ON \\
\hline 25 & Louise Temerty Breast Cancer Centre, Toronto, ON \\
\hline 26 & $\begin{array}{l}\text { Thunder Bay Regional Health Sciences Centre, Thunder Bay, } \\
\text { ON }\end{array}$ \\
\hline 27 & Trillium Health Partners, Mississauga, ON \\
\hline 28 & University of British Columbia, Victoria, BC \\
\hline 29 & William Osler Health System, Brampton, ON \\
\hline 30 & $\begin{array}{l}\text { Winnipeg Regional Health Authority, Breast Health Centre, } \\
\text { Winnipeg, MB }\end{array}$ \\
\hline 31 & Women's Breast Health Centre, Ottawa, ON \\
\hline 32 & Women's College Hospital, Toronto, ON \\
\hline 33 & Yukon Surgical Clinic, Whitehorse, YT \\
\hline
\end{tabular}

\section{Tumour Specimens}

Participants who consent to release their formalin-fixed, paraffin-embedded tumour blocks for future research have their tissue blocks sent to Women's College Hospital in Toronto for processing. Tissue microarrays are made after

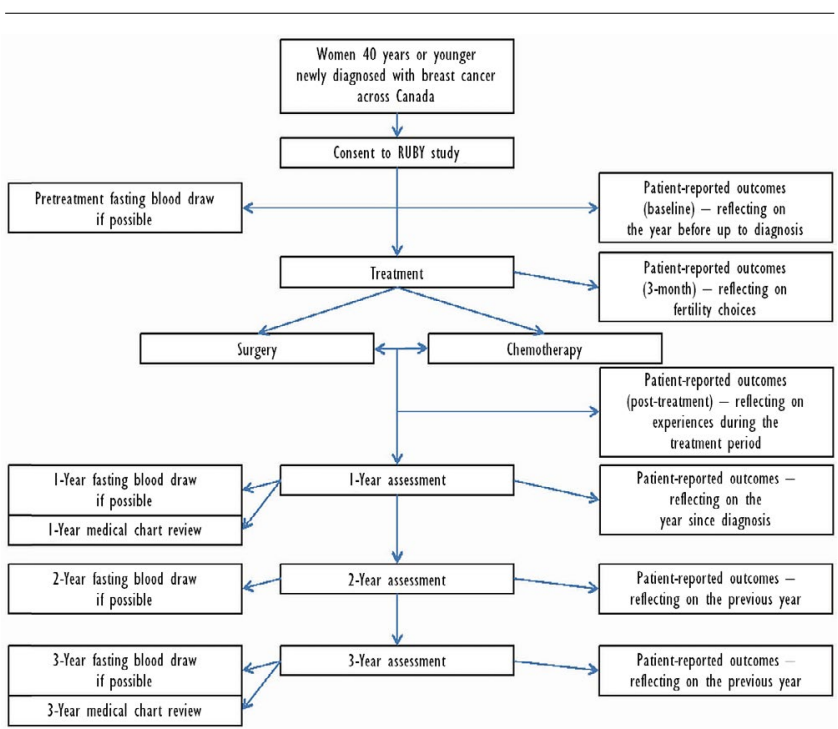

FIGURE 1 Patient flow through the study.

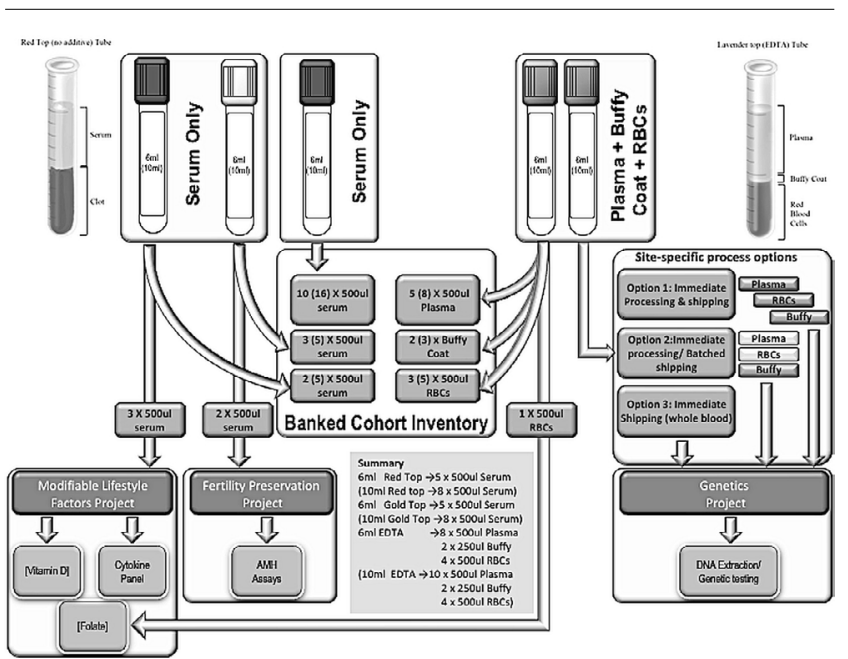

FIGURE 2 Process of collection and banking of serum, plasma, red blood cells, and buffy coats according to the standardized RUBY cohort study procedures. $\mathrm{RBCs}=$ red blood cells; $\mathrm{AMH}=$ anti-Müllerian hormone.

DNA and RNA are extracted from the tumour blocks. Sites with pathology capacity can also extract and create tissue microarrays directly for the RUBY biobank; the microarrays are then linked to clinical and outcomes data.

\section{Data Management}

Overall data management is conducted by the project team in Calgary using a central tracking database to monitor participant flow through the study and a unique RUBY identifier to link medical-record, patient-reported, and biospecimen data. The AHRC is the coordinating and data management centre responsible for receiving patient-reported and chart data. It has a dedicated project manager who liaises with the RUBY VRC, LRCs, and project team in Calgary. 


\section{Sample Size}

We estimated participant recruitment based on an annual projection of 1260 new cases of BCa in women 40 years of age and younger to be recruited between 2015 and 2020 . We estimate that our surgeon collaborators care for approximately $80 \%$ of new patients with вCa in Canada. We anticipate enrolling $30 \%-40 \%$ of those patients, (approximately 300 participants per year over 4 years) for a total enrolment of 1200 participants. At the time of writing we had anticipated recruitment to be completed by March 2020. That date has been affected by the COVID-19 pandemic.

\section{Patient and Public Involvement}

A research retreat of RUBY site leads, patient and family representatives, and community and patient advocates from Canadian agencies, including the Canadian Breast Cancer Network, was held in March 2017. A priority-setting partnership, inviting patients, family members, and caregivers to identify research priorities, was initiated in October 2019, with completion planned for the fall of 2020 .

\section{Ethics}

Ethics approval was obtained from each site's review boards and has been renewed as required. Separate ethics approval for each subproject has been obtained from the subproject lead's institution.

\section{RESULTS AT APRIL 2020}

\section{Recruitment Processes}

At April 2020, 33 recruitment sites had been activated (Table III). Each RUBY recruitment site has a surgeon lead working with $1-10$ other surgeons. Most centres $(n=22)$ are using LRCs; the others $(n=11)$ are using the VRC. That model permits recruitment at sites generally not accessible to traditional academic institution-based researchers, thus broadening the diversity of the cohort.

\section{Participants}

Recruitment started in July 2015. At 29 April 2020, 1161 participants had been recruited from 33 sites (Figure 3), for an average of 20 participants per month. Although it was projected that the planned sample size would be complete by March 2020 (Figure 4), the COVID-19 pandemic has affected recruitment timelines. At the time of writing, resumption of clinical services remains uncertain. The trial will continue to recruit after a 2-month suspension, but blood draws are on hold, and some sites are unable to provide support because of redeployment. We now estimate achieving our target by the end of 2020 .

Recruitment logs of eligible patients identified at each RUBY site were maintained from July 2015 until June 2017. During that time, $78.6 \%$ of eligible women were recruited. Themes reported as reasons for non-enrolment included patients being too overwhelmed, having no capacity to commit to the study, or not responding to repeated contact attempts after initially agreeing to be contacted. Calls with each RUBY surgeon principal investigator and the LRCs were held to troubleshoot location-specific barriers and to collaborate on potential solutions. In addition, monthly LRC

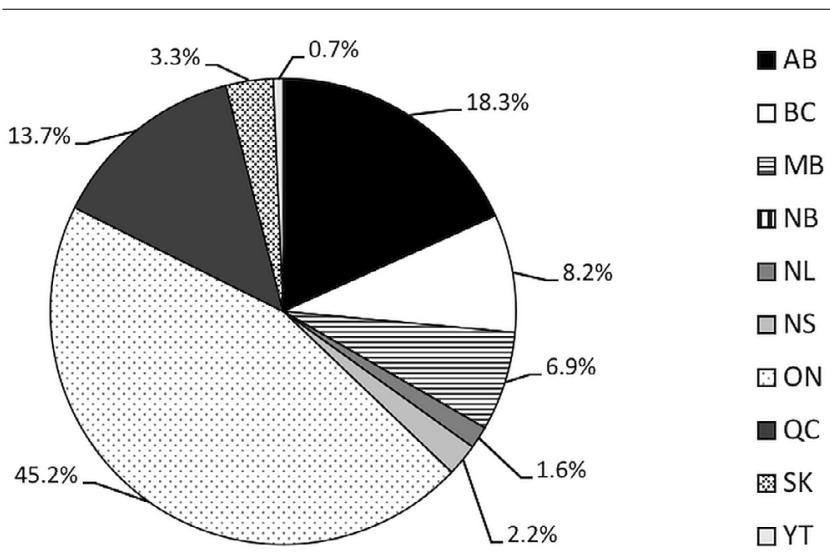

FIGURE 3 Geographic distribution of RUBY study participants based on province or territory of origin.

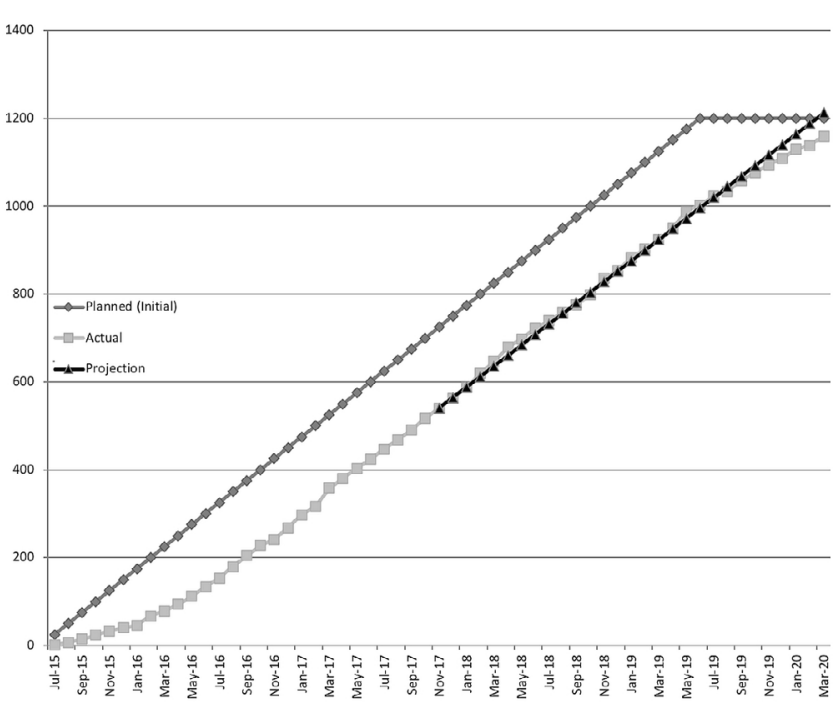

FIGURE 4 Recruitment progress (sample size plotted with initial projections). The planned sample size of 1200 participants had been anticipated to be achieved in March 2020.

and VRC teleconferences are held to provide important study updates and support from the central team, to obtain feedback from sites, and to foster engagement within the RUBY LRC community. To foster the RUBY network brand, quarterly RUBY newsletters are distributed digitally, highlighting RUBY achievements, recruitment sites, and personnel.

\section{RUBY Data Collection}

At April 2020, 81.4\% of the 1161 participants enrolled had agreed to all components of the study. Almost all agreed to provide biospecimens; 1080 of the 1161 (93.0\%) provided a baseline blood sample, and 1131 (97.4\%) gave consent for access to their formalin-fixed, paraffin-embedded tumour blocks. Table IV shows the proportion of participation in each study component. At April 2020, 89\% of the participants had responded to one or more of the questionnaires.

The consent for chart review, questionnaires, and blood samples permitted the objectives of 3 of the 4 
TABLE IV Study components completed by participants

\begin{tabular}{|c|c|c|c|c|c|}
\hline \multirow[t]{2}{*}{ Component } & \multicolumn{3}{|c|}{ Participants completing } & \multirow{2}{*}{$\begin{array}{c}\text { Sample } \\
\text { size }\end{array}$} & \multirow{2}{*}{$\begin{array}{l}\text { Percentage } \\
\text { completing }\end{array}$} \\
\hline & English & French & Total & & \\
\hline Questionnaires & 980 & 150 & 1130 & 1161 & 97.3 \\
\hline Blood samples & 935 & 145 & 1080 & 1161 & 93.0 \\
\hline FFPE samples & 985 & 146 & 1131 & $1160^{\mathrm{a}}$ & 97.5 \\
\hline Future contact & 870 & 139 & 1009 & 1161 & 86.9 \\
\hline
\end{tabular}

a When providing consent, 1 participant did not provide a response to this option.

FFPE $=$ formalin-fixed, paraffin-embedded.

subprojects to be embedded within the core RUBY processes. The 4 th subproject evaluating the association of germline mutations is consented separately. Participants in RUBY who consent for future research contact and who provide a blood sample at baseline are contacted 3 months after their initial surgical consultation. A specific consent outlining the risks and benefits associated with gene testing is obtained. Because of batched shipping of blood samples, participants and surgeons are informed that results are not timely for clinical care and should not replace standard clinical testing or usual practice. At April 2020, 858 patients had been contacted, and $580(68 \%)$ had consented to gene testing.

\section{Primary Tumour and Treatment Data}

At 29 April 2020, medical chart data for 610 patients had been entered. A revision of the Rave platform was performed in August 2019 in response to LRC feedback. Data validation for each patient chart is being performed by the core research team to ensure accuracy and to decrease missing data.

\section{SIGNIFICANCE AND IMPACT}

To date, we have successfully implemented-for an important and understudied oncology population-a national, comprehensive, and complex recruitment infrastructure that has achieved $97 \%$ of planned enrolment, with high levels of participation across all optional study domains, including patient-reported questionnaires, blood and tumour specimens, and consent for future contact. We have demonstrated the feasibility of recruiting patients outside traditional clinical trial environments in surgical offices, clinics, and centres, allowing for collection of data that starts at the time of diagnosis, which previously represented a gap in knowledge for patients with young-onset BCa.

Moreover, the creation of the RUBY network-consisting of site principal investigators who will act as knowledge translation vectors-will directly affect patient care and inform regional policies and programming for clinical care. To date, the RUBY network of surgeons has formally contributed to national вса surgery standards ${ }^{10}$ and consensus statements about contralateral prophylactic mastectomy ${ }^{11}$, and has had numerous informal communications and sharing of practices and experience.

The RUBY study will be the most comprehensive repository of data, biospecimens, and patient-reported outcomes ever collected for young women with BCa, which will enable productive research unique to this population now and in the future. This research model could be used for other oncology settings in Canada.

\section{ACKNOWLEDGMENTS}

This study is jointly funded by the Canadian Institutes of Health Research and the Canadian Breast Cancer Foundation-National (now Canadian Cancer Society-Research) 5-year grant competition for research about young women with BCa. Special thanks to Susan Isherwood PhD and Agota Ferenc MSc for project and data management in the creation of the cohort and manuscript.

\section{CONFLICT OF INTEREST DISCLOSURES}

We have read and understood Current Oncology's policy on disclosing conflicts of interest, and we declare that we have none.

\section{AUTHOR AFFILIATIONS}

*Department of Surgery, Cumming School of Medicine, ${ }^{\dagger}$ Department of Oncology, Cumming School of Medicine, and ${ }^{\ddagger}$ Department of Community Health Sciences, University of Calgary, Calgary, $\mathrm{AB} ;{ }^{\S}$ Department of Surgery, Li Ka Shing Knowledge Institute, St. Michael's Hospital, and "Women's College Hospital, University of Toronto, Toronto, ON; \#Department of Cancer Epidemiology and Prevention Research, CancerControl Alberta, Alberta Health Services, Calgary, AB; **Lawrence S. Bloomberg Faculty of Nursing, ${ }^{\dagger \dagger}$ Division of Medical Oncology, Sunnybrook Odette Cancer Centre, and ${ }^{\ddagger \ddagger}$ Applied Health Research Centre (AHRC), Li Ka Shing Knowledge Institute of St. Michael's Hospital, University of Toronto, Toronto, ON; ${ }^{\circledR \S} \mathrm{Clinical}$ Neurosciences Program, Alberta Health Services, Calgary, AB; \#\# Dalla Lana School of Public Health, University of Toronto, Toronto, ON.

\section{REFERENCES}

1. Canadian Cancer Statistics Advisory Committee. Canadian Cancer Statistics 2019. Toronto, ON: Canadian Cancer Society; 2019.

2. Voogd AC, Nielsen M, Peterse JL, et al. Differences in risk factors for local and distant recurrence after breast-conserving therapy or mastectomy for stage I and II breast cancer: pooled results of two large European randomized trials. JClin Oncol 2001;19:1688-97.

3. Rubino C, Arriagada R, Delaloge S, Le MG. Relation of risk of contralateral breast cancer to the interval since the first primary tumour. Br J Cancer 2010;102:213-19.

4. Narod SA. Breast cancer in young women. Nat Rev Clin Oncol 2012;9:460-70.

5. Copson E, Eccles B, Maishman T, et al. Prospective observational study of breast cancer treatment outcomes for U.K. women aged 18-40 years at diagnosis: the POsH study. J Natl Cancer Inst 2013;105:978-88.

6. Rosenberg SM, Sepucha K, Ruddy KJ, et al. Local therapy decision-making and contralateral prophylactic mastectomy 
in young women with early-stage breast cancer. Ann Surg Oncol 2015;22:3809-15.

7. Quan ML, Osman F, McCready D, Fernandes K, Sutradhar R, Paszat L. Postmastectomy radiation and recurrence patterns in breast cancer patients younger than age 35 years: a population-based cohort. Ann Surg Oncol 2014;21:395-400.

8. Bérubé S, Provencher L, Robert J, et al. Quantitative exploration of possible reasons for the recent improvement in breast cancer survival. Breast Cancer Res Treat 2007;106:419-31.
9. Cao JQ, Truong PT, Olivotto IA, et al. Should women younger than 40 years of age with invasive breast cancer have a mastectomy? 15-Year outcomes in a population-based cohort. Int J Radiat Oncol Biol Phys 2014;90:509-17.

10. Canadian Partnership Against Cancer (CPAC). Pan-Canadian Standards: Breast Cancer Surgery. Toronto, ON: CPAC; 2019.

11. Wright FC, Look Hong NJ, Quan ML, et al. Indications for contralateral prophylactic mastectomy: a consensus statement using modified Delphi methodology. Ann Surg 2018;267:271-9. 